\title{
Pretreatment SUV of the metastatic neck lymph nodes predicts neck control and survival in patients with stage IV oro/hypopharyngeal cancers
}

\author{
Abd El-Hafez, Y. ${ }^{*}$, Tzu-Chen, Y. ${ }^{* *}$, Khalil, H. ${ }^{* * *}$, Moustafa, $\mathbf{H}^{* * * *}$. \\ *Radiotherapy and Nuclear Medicine Department, South Egypt Cancer Institute, Assiut \\ University, Egypt, ${ }^{* *}$ Departments of Nuclear Medicine and Molecular Imaging Center, Chang \\ Gung Memorial Hospital and Chang Gung University, Taiwan, ${ }^{* * *}$ Oncology and Nuclear \\ Medicine Department, Faculty of Medicine, Cairo University, Egypt
}

\section{ABSTRACT}

Purpose To investigate the prognostic significance of standardized uptake value (SUV) of metastatic neck lymph nodes measured on FDG PET/CT in patients with stage IV oro/hypopharyngeal cancers treated by definitive chemoradiotherapy (CCRT).

Methods Retrospective analysis of 65 patients with clinically $\mathrm{N}+$ stage IV SCC of the oro/hypopharyngeal squamous cell carcinoma (SCC) who underwent FDG PET/CT scans for primary staging. Follow-up continued till death or at least 24 months from the start of treatment. The primary study endpoint was neck control (NC). The log-rank test and Cox proportional hazard analysis were used to identify significant prognostic factors.

Results The 3-year NC rate was 53\%. In univariate analysis, N3 status and nodal SUV $\geq$ 9.8 were significantly associated with reduced NC. In multivariable analyses, nodal SUV retained its independent prognostic significance as a predictor of NC. Lymph node stage was an independent predictor of disease specific survival (DSS). A prognostic scoring system was constructed as follows: score $0=\mathrm{N} 0-\mathrm{N} 2$ and nodal SUV $<9.8 ;$ score $1=\mathrm{N} 3$ or nodal SUV $\geq$ 9.8; and score $2=\mathrm{N} 3$ and nodal $\mathrm{SUV} \geq 9.8$. Patients with a score of 2 showed the worst NC (hazard ratio $[\mathrm{HR}], 95 \%$ confidence interval [CI]

\section{Correspondence author:}

\section{Dr. Yasser Abd El-Hafez}

Radiotherapy and Nuclear Medicine Department, South Egypt Cancer Institute, Assiut University, Egypt Email: yga1979@yahoo.com yga1979@gmail.com
$=10.5,3.3-33.1 ; P<0.001)$ and the lowest DSS (HR, 95\% CI = 6.4, 2.2-18.7; $P=0.001)$.

Conclusion The combination of high nodal SUV and N3 neck disease identifies a subgroup of high-risk stage IV oro/hypopharyngeal SCC patients. Further prospective studies are warranted to validate this finding.

\section{Keywords}

Neck lymph nodes - FDG PET SUV • Hypopharynx ' Oropharynx ' Prognosis . Concomitant chemoradiotherapy

\section{Introduction}

Metastasis to the neck lymph nodes predicts adverse outcomes in patients with head and neck cancers; even worse outcomes would be expected with regional treatment failure $[1,2]$. Definitive concurrent chemoradiotherapy is emerging as an alternative therapeutic approach for patients with advanced oro/hypopharyngeal cancers [3]; however, the necessity of planned neck dissection remains a controversial issue for patients with advanced neck stage ( $\geqq N 2)[4,5]$. Many established clinical and pathological features are important contributors to the development of neck recurrence [6]; yet, the investigation of pre-treatment clinicobiological predictors of neck control, as well as survival, remains an important issue. 
Research suggests that the standardized uptake value (SUV) - a semiquantitative measure of 18F-fluorodeoxyglucose (FDG) uptake on positron emission tomography (PET) - can predict tumor control and/or survival rates in patients with head and neck cancers [7-12]. However, most previous studies have focused on the prognostic value of primary tumor SUV, rather than of the neck lymph nodes [10, 13-16]. The tumor behaviors of metastatic nodes would be different from its original parent tumor cells, since it has gained genetic mutations of invasiveness and metastatic potential [17]. The aims of this study was to identify whether SUV at the neck lymph nodes is a significant prognostic factor in patients with stage IV oro/hypopharyngeal squamous cell carcinoma (SCC) treated by CCRT with curative intent.

\section{Materials and methods}

Study population

This retrospective study included a total of 65 patients, who presented to Chang Gung Memorial hospital between June 2006 and January 2009. All participants: (a) had a histologically-proven diagnosis of squamous cell carcinoma (SCC) of the oro/hypopharynx, (b) stage IV disease according to the 2002 American Joint Committee on Cancer (AJCC) staging criteria [18] with clinically positive neck nodes $(\mathrm{cN}+)$, (c) underwent PET/CT studies for primary staging, (d) completed the scheduled CCRT protocol, and (e) were followed-up for at least 24 months after the beginning of their primary definitive treatment or until death. We excluded patients with distant metastases at the time of diagnosis, synchronous primary tumors, or a previous history of head and neck cancer.

All patients had an extensive staging work-up, which included head and neck contrast-enhanced computed tomography (CE-CT) or magnetic resonance imaging (MRI), abdominal ultrasound (US). Questionable lesions were discussed jointly by our multidisciplinary head and neck team and solved by consensus. Echo-guided neck needle biopsy is indicated for inconsistent image findings. The study protocol was approved by the Institutional Review Board of the Chang Gung Memorial Hospital. All participants gave their written informed consent.

\section{${ }^{18}$ F-FDG PET/CT}

All patients were required to fast for at least 6 hours before PET imaging. Serum glucose levels were measured before the intravenous injection of $370 \mathrm{MBq}(10 \mathrm{mCi})$ of ${ }^{18} \mathrm{~F}-\mathrm{FDG}$. PET/CT imaging was performed with a modern hybrid PET/CT scanner (Discovery ST 16, GE Healthcare). Before PET acquisition, helical CT was performed from the head to the proximal thigh using a standardized protocol. The following settings were utilized: transverse $10-\mathrm{mm}$ collimation $\times 16$ modes, $120 \mathrm{kVp}$, auto $\mathrm{mAs}$ (range: 10-300), $0.5 \mathrm{~s}$ tube rotation, $17.5 \mathrm{~mm} / \mathrm{s}$ table speed, pitch 1.75. No oral or intravenous contrast agents were used. CT data were resized from a $512 \times 512$ matrix to a $128 \times 128$ matrix to match the PET data. Emission scans from the head to the proximal thigh were acquired at 50 min after injection of the tracer. Images were acquired in two-dimensional mode, 3 min per table position. PET images were reconstructed using CT for attenuation correction with the ordered-subset expectation maximization iterative reconstruction algorithm (4 iterations and 15 subsets). The maximum standardized uptake value of the neck lymph nodes (nodal SUVmax) was determined in all cases with PET-defined positive nodes. In the presence of multiple positive nodes, the highest SUV value was considered for the purpose of analysis. In PET-negative patients, a value of 2 was used to account for the typical head and neck background. Regions of interest (ROIs) were placed and measured over the lesions by PET on simultaneously displayed axial, coronal, and sagittal tomograms. The SUVmax was defined as the highest activity concentration per injected dose per body weight $(\mathrm{kg})$ after correction for radioactive decay.

Treatment and follow-up protocol

All patients were treated by primary CCRT with or without neoadjuvant chemotherapy. The drug scheme used in the majority of patients treated by neoadjuvant or concurrent chemotherapy consisted of cisplatin $50 \mathrm{mg} / \mathrm{m}^{2}$ biweekly plus daily oral 5-florouracil prodrug and leucovorin every 14 days [19]. All participants received intensity-modulated radiotherapy (IMRT) delivered through a 6-megavolt (MV) photon beam at 2 Gray (Gy) per fraction, with five fractions per week. The RT dose was 46-50 Gy for prophylaxis, and 70-76 Gy for the gross tumor and involved lymph nodes. 
Follow-up visits were scheduled every 1-2 months for the first 2 years, then every 3-4 months between the third and the fifth year. The tumor control status was assessed 3 months after ending treatment using different imaging modalities (PET/CT and MRI/CT) as well as by fiberoptic nasopharyngoscopy. Suspicious lesions were either biopsied or closely monitored. The diagnosis of recurrence was based either on positive histopathological findings or evidence of progression at follow-up imaging studies. If the patient has a resectable locoregional disease and with a good performance status, salvage surgery by curative intent will be evaluated. If salvage surgery is not amenable, palliative chemotherapy or best supportive care will be given.

Statistical analysis

The primary study endpoint was the 3-year neck control (NC) rate. Local control (LC), distant metastasis-free survival (DMFS), disease-specific (DSS) and overall survival (OS) served as secondary analyses. Survival times were calculated by Kaplan-Meier analysis and compared using the log-rank test. Cox proportional hazards models were used to identify independent prognostic factors. All time intervals were calculated from the beginning of treatment. We examined nodal SUVmax values (as a continuous variable) in relation to $\mathrm{N}$ status and other clinicopathological characteristics of the study participants using the Student's $t$-test for independent samples. The associations of nodal SUVmax (dichotomized according to the optimal cutoff value) with the study variables were examined using the $\chi^{2}$ test or Fisher's exact test. The optimal cutoff value for SUV was identified using the log-rank test based on the 3-year diseasespecific survival rate. All statistical analyses were performed with the SPSS package (version 15, SPSS Inc. Chicago, IL, USA). Values of $P<0.05$ (two-tailed) were considered statistically significant.

\section{Results}

Patient characteristics This study included 65 patients with oropharyngeal (OPC, $\mathrm{n}=28$ ) or hypopharyngeal (HPC, $\mathrm{n}=37$ ) SCC. The clinicopathological characteristics of the study participants are summarized in Table 1. At the end of the study, 33 patients died (3-year overall survival $=49 \%)$ and 26 had neck failure $(3$-year NC $=53 \%)$. Isolated neck failure was noted in 12 patients (46\%). Neck failure was associated with local failure in 5 (19\%), with distant failure in 2 $(8 \%)$, and with both local and distant failures in the remaining 7 patients (27\%). LC, DMFS, DSS and OS were 62, 70, 59 and 49\%, respectively. Nodal SUV cut-off value $9.8 \mathrm{~g} / \mathrm{mL}$ was identified as the best cut-off for 3-year DSS.

Table 1 Clinicopathological characteristics of the study participants $(n=65)$

\begin{tabular}{lll} 
Parameter & $n$ & $\%$ \\
\hline TNM Stage & & \\
\hline IV-A & 40 & $(61.5)$ \\
IV-B & 25 & $(38.5)$ \\
T status & & \\
T1 & 5 & $(7.7)$ \\
T2 & 11 & $(16.9)$ \\
T3 & 7 & $(10.8)$ \\
T4a & 22 & $(33.8)$ \\
T4b & 20 & $(30.8)$ \\
N status & & \\
N1 & 3 & $(4.6)$ \\
N2b & 29 & $(44.6)$ \\
N2c & 22 & $(33.8)$ \\
N3 & 11 & $(16.9)$ \\
Differentiation & & \\
Well-differentiated & 1 & $(1.5)$ \\
Moderately-differentiated & 42 & $(64.6)$ \\
Poorly-differentiated & 11 & $(16.9)$ \\
Unknown & 11 & $(16.9)$ \\
A & &
\end{tabular}

Age, years

Median (range) ${ }^{a}$

54

(36-74)

Radiotherapy dose

Median Gy (Range) ${ }^{\text {a }}$

72

$(66-90)$

SUV $_{\text {max }}$ of the neck lymph nodes

Median (Range) ${ }^{a}$

10.1

$(2.0-25.7)$

${ }^{a}$ Data in parenthesis indicate the range

\section{Nodal SUV associations with other study variables}

Nodal SUV (analyzed as a continuous variable) was signifycantly higher in N3 patients (mean $=15.3 \pm$ 5.7) than $\mathrm{N} 1$ (mean $\pm \mathrm{SD}=5.3 \pm 1.4 ; P=0.02$ ) and $\mathrm{N} 2$ (mean $\pm \mathrm{SD}=10 \pm 5.4 ; P=0.01$ ). Similarly, high nodal SUV values $(\geq 9.8 \mathrm{mg} / \mathrm{mL})$ were significantly associated with nodal status. Ten out of 11 patients with $\mathrm{N} 3$ had nodal $\mathrm{SUV} \geq 9.8(P=$ 0.02). No other associations between the nodal SUV and other parameters were observed. 


\section{Univariate analysis}

Univariate analysis for neck events demonstrated a significantly lower $\mathrm{NC}$ rate for patients with high nodal SUV (26 vs. 81\%; $P<0.001$ ) or N3 neck disease (25 vs. 76\%; $P=0.003$ ), and tended to be lower with stage IV-b disease (41 vs. $60 \% ; P=$ $0.056)$. Further, betel quid chewing was associated with lower NC rate, albeit not significantly so.
Patients with high nodal SUV values or N3 disease had worse disease-specific and overall survival rates compared with those who do not have these risk factors (Table 2). In addition, the overall survival rate was lower in patients with HPC than in those with OPC

(38vs. 63\%; $P=0.027$ ).

Table 2 Univariate analysis of 3-year local control (LC), neck control (NC), distant metastasis-free survival (DMFS), disease-specific survival (DSS), and overall survival (OS) rates in 65 patients with stage IV oro/hypopharyngeal cancer

\begin{tabular}{|c|c|c|c|c|c|c|c|c|c|c|c|}
\hline \multirow{2}{*}{ Risk factor } & \multirow{2}{*}{$\mathbf{n}$} & \multicolumn{2}{|l|}{ LC } & \multirow{2}{*}{$\begin{array}{c}\mathrm{NC} \\
\text { (Events, \%) }\end{array}$} & \multicolumn{3}{|c|}{ DMFS } & \multirow{2}{*}{$\begin{array}{c}\text { DSS } \\
\text { (Events, \%) }\end{array}$} & \multicolumn{3}{|c|}{ OS } \\
\hline & & (Events,\%) & $P$ & & $P$ & (Events, \%) & $P$ & & $P$ & (Events, \%) & $P$ \\
\hline \multicolumn{12}{|l|}{ Cancer site } \\
\hline Oropharynx & 28 & $(4,83)$ & $0.014 *$ & $(9,64)$ & 0.141 & $(3,87)$ & $0.041 *$ & $(9,68)$ & 0.238 & $(10,63)$ & $0.027 *$ \\
\hline Hypopharynx & 37 & $(15,42)$ & & $(17,43)$ & & $(10,54)$ & & $(15,52)$ & & $(23,38)$ & \\
\hline \multicolumn{12}{|l|}{ Differentiation } \\
\hline WD-MD & 43 & $(12,63)$ & 0.816 & $(16,57)$ & 0.533 & $(6,77)$ & 0.566 & $(13,66)$ & 0.638 & $(20,53)$ & 0.623 \\
\hline PD SCC & 11 & $(3,64)$ & & $(5,40)$ & & $(2,74)$ & & $(4,61)$ & & $(6,45)$ & \\
\hline \multicolumn{12}{|l|}{ Stage } \\
\hline IVa & 40 & $(11,66)$ & 0.371 & $(13,60)$ & 0.056 & $(6,78)$ & 0.087 & $(10,72)$ & 0.011* & $(17,57)$ & 0.069 \\
\hline IVb & 25 & $(8,55)$ & & $(13,41)$ & & $(7,57)$ & & $(14,40)$ & & $(16,36)$ & \\
\hline \multicolumn{12}{|l|}{ T status } \\
\hline T1-T3 & 23 & $(3,79)$ & $0.040 *$ & $(11,46)$ & 0.751 & $(4,73)$ & 0.529 & $(6,70)$ & 0.175 & $(10,55)$ & 0.338 \\
\hline $\mathbf{T 4}$ & 42 & $(16,52)$ & & $(15,58)$ & & $(9,69)$ & & $(18,54)$ & & $(23,45)$ & \\
\hline \multicolumn{12}{|l|}{ N status } \\
\hline N1-N2 & 54 & $(14,67)$ & $0.044 *$ & $(18,59)$ & 0.003* & $(9,76)$ & 0.018* & $(16,67)$ & $0.005^{*}$ & $(24,55)$ & $0.017 *$ \\
\hline $\mathbf{N 3}$ & 11 & $(5,24)$ & & $(8,22)$ & & $(4,25)$ & & $(8,21)$ & & $(9,18)$ & \\
\hline \multicolumn{12}{|l|}{ Treatment } \\
\hline CCRT & 36 & $(9,70)$ & 0.596 & $(12,59)$ & 0.474 & $(5,81)$ & 0.263 & $(14,57)$ & 0.518 & $(19,46)$ & 0.509 \\
\hline $\mathrm{C} / \mathrm{T}+\mathrm{CCRT}$ & 29 & $(10,50)$ & & $(14,46)$ & & $(8,58)$ & & $(10,62)$ & & $(14,52)$ & \\
\hline \multicolumn{12}{|l|}{ Nodal SUV } \\
\hline$<9.8$ & 29 & $(7,73)$ & 0.065 & $(5,81)$ & $\begin{array}{l}< \\
0.001 *\end{array}$ & $(4,83)$ & $\mathbf{0 . 0 3 3}^{*}$ & $(6,78)$ & $0.006^{*}$ & $(9,69)$ & $\mathrm{0.003}^{*}$ \\
\hline$\geq 9.8$ & 36 & $(12,48)$ & & $(21,26)$ & & $(9,53)$ & & $(18,43)$ & & $(24,32)$ & \\
\hline
\end{tabular}

WD/MD/PD well/moderately/poor differentiated, SCC squamous cell carcinoma, CCRT concomitant chemoradiotherapy, C/T neoadjuvant chemotherapy, SUV standardized uptake value

- Indicates significant results $(\mathrm{P}<0.05)$

\section{Multivariate analysis and prognostic scoring} system

All variables with $P<0.10$ in univariate analysis were tested in a Cox proportional hazard model. (Table 3) shows the results of multivariable Cox regression analysis. High nodal SUV $\geq 9.8$ and N3 neck status were the only two variables to retain their independent prognostic significance 
for NC and DSS after allowance for potential confounders. Based on the presence or absence of the two independent variables, we constructed a prognostic scoring system that identified three distinct prognostic subgroups (Table 4), as follows: score $0=\mathrm{N} 0-\mathrm{N} 2$ and nodal SUV $<9.8$; score $1=\mathrm{N} 3$ or nodal SUV $\geq 9.8$; and score $2=$
$\mathrm{N} 3$ and nodal SUV $\geq 9.8$. Cox regression analysis, corrected for both $\mathrm{T}$ status and cancer site, showed that patients with a score of 2 experienced the worst outcomes in terms of $\mathrm{NC}$ (HR, 95\% CI = 13.9, 3.1-62.8; $P=0.001)$ and DSS (HR, 95\% CI $=6.7,1.9-23.6 ; P=0.003)$ as well as other endpoints (Figure 1), (Table 5).

Table 3 Multivariable analyses of 3-year local control (LC), neck control (NC), distant metastasisfree survival (DMFS), disease-specific survival (DSS), and overall survival (OS) rates in 65 patients with stage IV oro/hypopharyngeal cancer

\begin{tabular}{lllll}
\hline \multirow{2}{*}{ Outcome } & \multicolumn{2}{c}{$\begin{array}{c}\text { N status (N1-N2/N3) } \\
(\mathrm{n}=54 / 11)\end{array}$} & \multicolumn{2}{c}{$\begin{array}{c}\text { Nodal SUV }(<9.8 />9.8) \\
(\mathrm{n}=30 / 35)\end{array}$} \\
\cline { 2 - 5 } & HR $(95 \% \mathrm{CI})$ & $P$ & HR $(95 \%$ CI $)$ & $P$ \\
\hline LC & $1.7(0.6-5)$ & 0.356 & $2.8(1-7.8)$ & 0.056 \\
NC & $2.2(0.9-5.5)$ & 0.100 & $4.9(1.7-14)$ & $0.003 *$ \\
DMFS & $3.1(0.9-11.1)$ & 0.080 & $2.8(0.8-10.4)$ & 0.116 \\
DSS & $2.6(1-6.5)$ & $0.048 *$ & $3.1(1.1-8.4)$ & $0.026 *$ \\
OS & $1.9(0.8-4.4)$ & 0.129 & $2.7(1.2-6)$ & $0.020 *$ \\
\hline
\end{tabular}

$H R$ hazard ratio (stepwise forward method), $C I$ confidence interval, $S U V$ standardized uptake value

* Indicates significant results, results corrected for T status and tumor site

Table 4 Cox regression analysis of 3-year local conrol (LC), neck control (NC), distant metastasis-free survival (DMFS), disease-specific survival (DSS), and overall survival (OS) rates in 65 patients with stage IV oro/hypopharyngeal cancer according to the prognostic risk score

\begin{tabular}{|c|c|c|c|c|c|c|c|c|c|}
\hline \multirow{2}{*}{ Outcome } & \multicolumn{3}{|c|}{ Score $0(n=28)^{a}$} & \multicolumn{3}{|c|}{ Score $1(n=27)$} & \multicolumn{3}{|c|}{ Score $2(n=10)$} \\
\hline & $\begin{array}{l}\text { (Events, } \\
\%)\end{array}$ & $\begin{array}{l}\text { HR }(95 \% \\
\text { CI) }\end{array}$ & $P_{0}$ & $\begin{array}{l}\text { (Events, } \\
\%)\end{array}$ & $\begin{array}{l}\text { HR }(95 \% \\
\text { CI) }\end{array}$ & $P_{1}$ & $\begin{array}{l}\text { (Events, } \\
\%)\end{array}$ & $\begin{array}{l}\text { HR } \\
(\mathbf{9 5 \%} \mathrm{CI})\end{array}$ & $P_{2}$ \\
\hline LC & $(6,76)$ & 1 & 0.104 & $(9,47)$ & $2.2(0.7-6.4)$ & 0.166 & $(4,39)$ & $3.9(1.1-14.3)$ & $0.038 *$ \\
\hline $\mathrm{NC}$ & $(5,80)$ & 1 & $<0.001$ & $(13,36)$ & $3.3(1.1-9.7)$ & 0.029* & $(8,13)$ & $10.5(3.3-33.1)$ & $<0.001 *$ \\
\hline DMFS & $(3,87)$ & 1 & 0.044 & $(7,51)$ & $3(0.7-12.2)$ & 0.122 & $(3,37)$ & $8.4(1.6-45.2)$ & $0.013 *$ \\
\hline DSS & $(6,77)$ & 1 & 0.003 & $(10,58)$ & $2.4(0.8-6.8)$ & 0.101 & $(8,11)$ & $6.4(2.2-18.7)$ & $0.001 *$ \\
\hline OS & $(9,68)$ & 1 & 0.004 & $(15,42)$ & $2.1(0.9-5)$ & 0.086 & $(9,10)$ & $4.9(1.9-12.5)$ & $0.001 *$ \\
\hline
\end{tabular}

$H R$ hazard ration, $C I$ confidence interval, $S U V$ standardized uptake value

${ }^{a}$ Score 0 (N0-N2 \& nodal SUV $<9.8$, reference group), score 1 (N3 or nodal SUV $\geq 9.8$ ), score 2 (N3 \& nodal $\mathrm{SUV} \geq 9.8$ )

$P_{0}$ represents the within-groups difference, $P_{1} \& P_{2}$ represents the difference between score $1 \&$ score 2 to the reference group respectively

- Indicates significant results, results were corrected for tumor site and $\mathrm{T}$ stage 
Table 5 Stratification of N1-N2 patients by nodal SUV according to 3-year local control (LC), neck control (NC), distant metastasis-free survival (DMFS), disease-specific survival (DSS), and overall survival (OS) rates

\begin{tabular}{|c|c|c|c|}
\hline \multirow[t]{2}{*}{ Outcome } & $\begin{array}{c}\text { Nodal SUV }<9.8 \\
(n=28)\end{array}$ & $\begin{array}{c}\text { Nodal } S U V \geq 9.8 \\
(n=26)\end{array}$ & \multirow{2}{*}{$P$} \\
\hline & (Events, \%) & (Events, \%) & \\
\hline $\mathbf{L C}$ & $(6,76)$ & $(8,53)$ & 0.141 \\
\hline $\mathrm{NC}$ & $(5,80)$ & $(13,32)$ & $0.002 *$ \\
\hline DMFS & $(3,87)$ & $(6,57)$ & 0.062 \\
\hline DSS & $(6,77)$ & $(10,56)$ & 0.092 \\
\hline OS & $(9,68)$ & $(15,40)$ & $0.034 *$ \\
\hline
\end{tabular}

SUV standardized uptake value

- Indicates significant results $(P<0.05)$
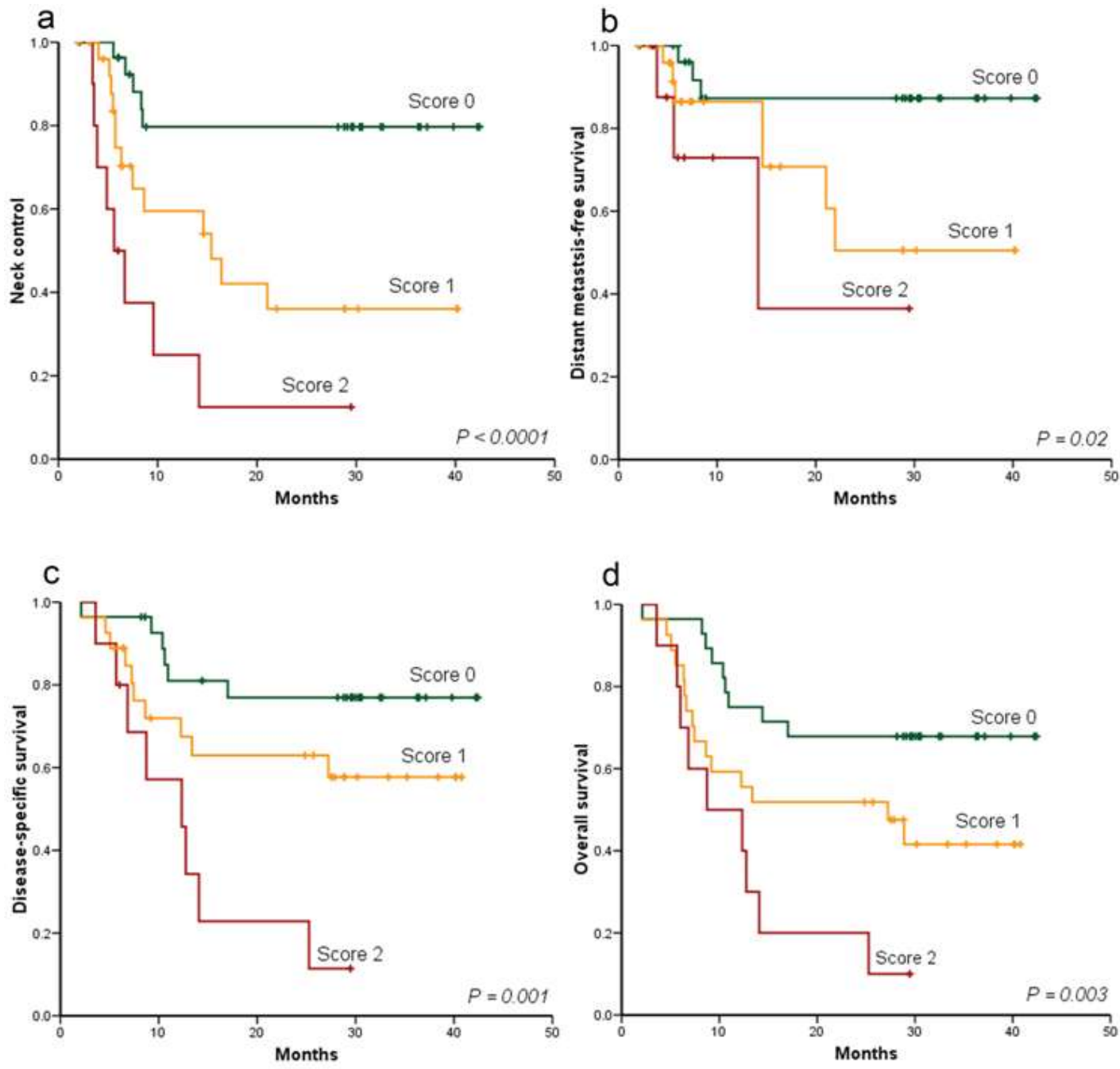

Fig. 1 Neck control (a), distant metastasis free survival (b), disease-specific survival (c) and overall survival (d) according to the proposed risk score ${ }^{a}$ in 65 patients with stage IV oro/hypopharyngeal cancer ${ }^{a}$ Score 0 (N0-N2 \& nodal SUV < 9.8), score 1 (N3 or nodal SUV $\geq 9.8$ ), score 2 (N3 \& nodal SUV $\geq 9.8$ ) $P$ value obtained from log-rank test 
The oro/hypopharynx harbors a rich lymphatic network, and more than $60 \%$ of cancers arising in this area present in advanced stage [20]. The regional response to therapy is an established prognostic factor for oro/hypopharyngeal squamous cell carcinomas and the identification of novel predictors of neck control may be useful for individualizing treatment approaches.

The FDG uptake in the primary tumor (as measured by the SUV) has been shown to correlate with both tumor proliferation rates [21] and clinical outcomes [7-12] in head and neck cancers. Although the potential prognostic significance of nodal SUV values at oral cavity cancer and some other cancers has been reported $[14-16,22,23]$, a paucity of data exists on the potential prognostic significance of nodal SUV values in oro/hypopharyngeal cancer.

Herein we have shown that a nodal SUV of 9.8 or higher may independently predict the 3-year NC and survival rates in stage IV oro/hypopharyngeal cancer patients treated by CCRT with curative intent. Importantly, we have developed a prognostic scoring system that incorporates nodal SUV values and identifies three distinct prognostic subgroups.

Patients with a score of 2 showed the highest rates of neck and distant failures, cancer deaths, and poor overall survival. Seventy percent of patients with a score of 2 died of disease within 15 months after the beginning of CCRT. Although our results cannot be presented as practice-changing, the poor prognosis within this group may prompt further studies to evaluate the potential usefulness of novel approaches such as early salvage surgery for resectable disease, more effective chemotherapy regimens, radiotherapy dose escalation or biological therapies.

Patients with score 0 showed the lowest rate of distant failures (3/28) when compared to score 1 $(7 / 27)$ and score $2(3 / 10)$. DMFS was $87 \%$ for score $0,51 \%$ for score $1(P=0.03)$ and $37 \%$ for score $2(P=0.02)$. Closely systemic workup may be advised for early detection of distant metastases in patients with score 1 or 2 .

In our data, 10 of 11 patients with N3 disease had nodal SUV $\geq 9.8(P=0.02)$. Although we found that strong association between $\mathrm{N} 3$ and high nodal SUV, but that does not reduce -in our opinion- the prognostic importance of nodal SUV. When we stratify N1-N2 patients by the nodal SUV (Table 5 ) we found strong relation to unfavorable outcomes in patients with high nodal SUV; neck control and overall survival were significantly lower, while the distant failure and disease specific survival remained marginally significant. Eight patients in this category were successfully salvaged by neck dissection. There might be a potential role of early planned neck dissection for such patient group, who has N1-2 stage but with higher nodal SUV. Further prospective investigation trial is warranted.

We recognize that our study has several limitations. The single-center nature and the retrospective design of our research limit the generalizability of our findings. Second, we did not specifically investigate the accuracy of FDG-PET in the diagnosis of nodal disease. Not with standing these caveats, the potential strengths of our study include the homogenous study population and the use of standardized treatment and follow-up protocols.

\section{Conclusions}

Our study suggests that the combination of high nodal SUV and N3 neck disease identifies a subgroup of high-risk stage IV oro/hypopharyngeal SCC patients for both regional recurrence and survival. Further prospective studies are warranted.

\section{Acknowledgements}

This study was supported by grants CMRPG360071, CMRPG360072 and CMRPG360073 from the Chang Gung Memorial Hospital.

\section{Conflicts of interest}

The authors do not have anything to disclose

\section{References}

1.Layland MK, Sessions DG, Lenox J. The influence of lymph node metastasis in the treatment of squamous cell carcinoma of the oral cavity, oropharynx, larynx, and hypopharynx: N0 versus N+. Laryngoscope. 115:629-39; 2005.

2.Lindelv B, Hansen HS. The impact of lymph node metastases on the results of treatment by primary radiotherapy and secondary surgery in oropharyngeal cancer. Acta Oncol. 34:965-8; 1995.

3.Wong SJ, Harari PM, Garden AS, Schwartz M, Bellm L, Chen A, et al. Longitudinal oncology registry of head and neck carcinoma (LORHAN): analysis of chemoradiation treatment approaches in the United States. Cancer. 2010. 
4.Lango MN, Myers JN, Garden AS. Controversies in surgical management of the node-positive neck after chemoradiation. Semin Radiat Oncol. 19:24-8; 2009.

5.Pellitteri PK, Ferlito A, Rinaldo A, Shah JP, Weber RS, Lowry J, et al. Planned neck dissection following chemoradiotherapy for advanced head and neck cancer: is it necessary for all? Head Neck. 28:166-75; 2006.

6.Varghese C, Sankaranarayanan R, Nair B, Nair $\mathrm{MK}$. Predictors of neck node control in radically irradiated squamous cell carcinoma of the oropharynx and laryngopharynx. Head Neck. 15:105-8; 1993.

7.Allal AS, Dulguerov P, Allaoua M, Haenggeli CA, El-Ghazi el A, Lehmann W, et al. Standardized uptake value of 2-[(18)F] fluoro-2deoxy-D-glucose in predicting outcome in head and neck carcinomas treated by radiotherapy with or without chemotherapy. J Clin Oncol. 20:1398404; 2002.

8.Halfpenny W, Hain SF, Biassoni L, Maisey MN, Sherman JA, McGurk M. FDG-PET. A possible prognostic factor in head and neck cancer. $\mathrm{Br} \mathrm{J}$ Cancer. 86:512-6; 2002.

9.Minn H, Lapela M, Klemi PJ, Grenman R, Leskinen S, Lindholm $P$, et al. Prediction of survival with fluorine-18-fluoro-deoxyglucose and PET in head and neck cancer. $J$ Nucl Med. 38:1907-11; 1997.

10.Schwartz DL, Rajendran J, Yueh B, Coltrera MD, Leblanc M, Eary J, et al. FDG-PET prediction of head and neck squamous cell cancer outcomes. Arch Otolaryngol Head Neck Surg. 130:1361-7, 2004.

11.Torizuka T, Tanizaki Y, Kanno T, Futatsubashi M, Naitou K, Ueda Y, et al. Prognostic value of 18F-FDG PET in patients with head and neck squamous cell cancer. AJR Am J Roentgenol. 192:W156-60; 2009.

12.Wong RJ, Lin DT, Schoder H, Patel SG, Gonen M, Wolden S, et al. Diagnostic and prognostic value of $[(18)$ F]fluorodeoxyglucose positron emission tomography for recurrent head and neck squamous cell carcinoma. J Clin Oncol. 20:4199-208; 2002.

13.Kubicek GJ, Champ C, Fogh S, Wang F,
Reddy E, Intenzo C, et al. FDG-PET staging and importance of lymph node SUV in head and neck cancer. Head Neck Oncol. 2:19; 2010.

14.Liao CT, Chang JT, Wang HM, Ng SH, Hsueh C, Lee LY, et al. Preoperative [18F]fluorodeoxyglucose positron emission tomography standardized uptake value of neck lymph nodes predicts neck cancer control and survival rates in patients with oral cavity squamous cell carcinoma and pathologically positive lymph nodes. Int $\mathbf{J}$ Radiat Oncol Biol Phys. 74:1054-61; 2009.

15.Liao CT, Chang JT, Wang HM, Ng SH, Huang $\mathrm{SF}$, Chen IH, et al. Preoperative [18F]fluorodeoxyglucose positron emission tomography standardized uptake value of neck lymph nodes may aid in selecting patients with oral cavity squamous cell carcinoma for salvage therapy after relapse. Eur J Nucl Med Mol Imaging. 36:1783-93; 2009.

16.Liao CT, Wang HM, Chang JT, Lin CY, Ng SH, Huang SF, et al. Influence of pathological nodal status and maximal standardized uptake value of the primary tumor and regional lymph nodes on treatment plans in patients with advanced oral cavity squamous cell carcinoma. Int J Radiat Oncol Biol Phys. 77:421-9; 2010.

17.Chiang AC, Massague J. Molecular basis of metastasis. N Engl J Med. 359:2814-23; 2008.

18.Greene FL, American Joint Committee on Cancer., American Cancer Society. AJCC cancer staging manual. 6th ed. New York: SpringerVerlag; 2002.

19.Wang HM, Wang CS, Chen JS, Chen IH, Liao CT, Chang TC. Cisplatin, tegafur, and leucovorin: a moderately effective and minimally toxic outpatient neoadjuvant chemotherapy for locally advanced squamous cell carcinoma of the head and neck. Cancer. 94:2989-95; 2002.

20.Brady LW, Connor NP, Grau C, Harari PM, Heilmann HP, Molls M, et al. Functional Preservation and Quality of Life in Head and Neck Radiotherapy. In: Medical Radiology,. Springer Berlin Heidelberg. 1999.

21.Haberkorn U, Strauss LG, Reisser C, Haag D, Dimitrakopoulou A, Ziegler S, et al. Glucose uptake, perfusion, and cell proliferation in head and neck tumors: relation of positron emission tomography to flow cytometry. J Nucl Med. 32:1548-55; 1991. 
22. Okereke IC, Gangadharan SP, Kent MS, Nicotera SP, Shen C, DeCamp MM. Standard uptake value predicts survival in non-small cell lung cancer. Ann Thorac Surg. 88:911-5 ; discussion 5-6; 2009.
23.Yen TC, See LC, Lai CH, Tsai CS, Chao A, Hsueh $S$, et al. Standardized uptake value in paraaortic lymph nodes is a significant prognostic factor in patients with primary advanced squamous cervical cancer. Eur $\mathrm{J}$ Nucl Med Mol Imaging. $35: 493-501 ; 2008$. 